\title{
Actions et effets secondaires de l'abus de Stéroïdes Anabolisants dans le sport et la vie sociale
}

\author{
Alan James GEORGE \\ Liverpool John Moores University, London
}

\section{RESUME}

Les stéroïdes anabolisants (SA) dérivés de la testostérone ont à la fois des effets anabolisants (augmentation du muscle et de la force) et androgéniques (caractères sexuels primaires et secondaires). Les efforts entrepris pour limiter les effets androgéniques tout en augmentant les effets anabolisants n'ont pas été couronnés de succès. Les altérations de la structure de la testostérone, induites pour augmenter la pharmacocinétique des SA, ont donné naissances à des produits pharmaceutiques qui sont actifs par voie orale, ont une demivie plasmatique accrue et peuvent être administrés sous forme d'injections retards. Des doses thérapeutiques de SA produisent des effets statistiquement significatifs sur la force et la performance athlétique dans des essais cliniques et scientifiquement bien contrôlés. A faibles doses thérapeutiques, l'alimentation et un entraînement intensif sont également importants pour produire une augmentation statistiquement significative de la force. Des doses supérieures à 6 à $7000 \mathrm{mg}$ par semaine sont régulièrement utilisées dans le sport et produisent la plus grande augmentation de la force musculaire, de l'érythropoïèse et de la masse corporelle maigre. Les profils d'utilisation abusive de stéroïdes peuvent être complexes, reflétant un désir de minimiser les effets secondaires et d'échapper à la détection. Les effets secondaires des stéroïdes anabolisants sont de plusieurs types. Les SA augmentent la rétention d'eau et de sel, conduisant à une expansion du volume sanguin, mais les effets des stéroïdes sur la pression artérielle sont équivoques et la plupart des effets cardiovasculaires secondaires semblent être réversibles.

L'abus de SA entraîne une augmentation des taux sanguins de triglycérides et de cholestérol, et ceci est associé à une diminution des Lipoprotéines de Haute Densité (HDL) et à une augmentation des Lipoprotéines de Faible Densité (LDL). Bien que ces effets soient réversibles, ils sont associés à un risque accru de pathologie cardiovasculaire à la fois aiguë et chronique. Les effets secondaires irréversibles les plus sérieux des stéroïdes anabolisants concernent des cancers, principalement du foie, de la prostate et du rein. Les carcinomes hépatiques sont fortement liés à l'abus des formes actives de stéroïdes substituées en 17-alphaméthyl qui provoquent aussi une jaunisse réversible. Chez les hommes, l'abus de stéroïdes anabolisants entraîne la suppression de la libération de LH et FSH conduisant à une inhibition de la production de testostérone souvent accompagnée d'une atrophie testiculaire, et à une azoospermie. Des doses élevées et régulières de produits peuvent aussi causer des effets, modérés à sévères, de féminisation sous la forme de gynécomastie. L'apparition de caractères sexuels secondaires de type masculin est un effet secondaire de l'abus de SA chez les femmes. Une résistance augmentée à l'insuline et une hyperglycémie à jeun constituent les effets secondaires non gonadiques les plus courants des SA.

L'abus de SA amène des modifications comportementales et psychiatriques contradictoires et complexes. On a rapporté une augmentation de la fréquence des maladies mentales chez les utilisateurs abusifs de stéroïdes anabolisants, incluant la schizophrénie paranoïde, la manie et la dépression.

\footnotetext{
Correspondance :

Dr Alan James George - Reader in Clinical Pharmacology \& Director of Clinical Research Courses, School of Pharmacy and Chemistry, Liverpool John Moores University, Byrom Street, Liverpool, L3 3AF. U.K.
} 
Une dépendance physique et psychologique survient chez certains utilisateurs abusifs, et des troubles psychiatriques sévères peuvent apparaître lors du sevrage, conduisant dans quelques cas à la criminalité et même au suicide. Des études plus nombreuses sont nécessaires quant aux effets à long terme des SA. Les implications de l'abus de SA pendant les $\mathbf{5 0}$ dernières années sont discutées dans la revue.

Mots clés : stéroïdes anabolisants, stéroïdes anabolisants androgéniques, testostérone, effets secondaires, sport, athlètes, dépendance, revue, réversibilité, chronique

\section{INTRODUCTION}

Le légendaire Zauberlehrling (l'apprenti sorcier) de Goethe jette un sort pour économiser du temps et de l'effort en l'absence de son maître, mais il découvre que l'enchantement ne peut pas être rompu. Les promoteurs d'un abus des stéroïdes anabolisants sont dans une semblable position.

La testostérone, dont dérivent les stéroïdes anabolisants, a été isolée pour la première fois en 1935, et ses dérivés synthétiques furent commercialisés à partir de 1945. On présume que les derniers Jeux Olympiques sans stéroïdes ont été les Jeux Olympiques controversés de Berlin en 1936 [25].

En dépit de nombreuses rumeurs, il n'existe aucune preuve que soit de la testostérone soit des stéroïdes aient été administrés aux régiments de Nazi Waffen SS pendant la seconde guerre mondiale [86]. La première apparition documentée des stéroïdes anabolisants dans un événement sportif majeur fut lors des Championnats du Monde d'Haltèrophilie à Vienne en 1954 [86].

Depuis les années 1950, la chimie et la pharmacologie médicinales des stéroïdes anabolisants ont fait des progrès $[42,78]$.

Les stéroïdes anabolisants provenant de la testostérone possèdent à la fois des effets anaboliques (augmentant le muscle et la puissance) et androgéniques (caractères sexuels primaires et secondaires), et les efforts importants menés pour limiter les effets androgéniques tout en augmentant les effets anabolisants n'ont pas été fructueux. Les modifications de la structure de la testostérone réalisées dans le but d'améliorer la pharmacocinétique des stéroïdes anabolisants ont donné naissance à des produits pharmaceutiques qui sont actifs par voie orale, ont une demi-vie plasmatique plus longue, et peuvent être administrés sous forme d'injections retards. Les stéroïdes anabolisants actifs par voie orale sont substitués en position 17-alpha-méthyl, ce qui augmente aussi leur toxicité hépatique, alors que l'estérification par une longue chaîne d'acide carboxylique permet l'utilisation des stéroïdes anabolisants sous forme de substance retard $[25,42,49]$.

Les doses thérapeutiques de stéroïdes anabolisants sont de 2,5 à 10 mg par jour au Royaume Uni, et des effets positifs, statistiquement significatifs, sur la puissance et la performance athlétique ont été obtenus en utilisant ces doses dans des essais cliniques bien contrôlés au plan scientifique. A ces faibles doses, «éthiques» et thérapeutiques, l'alimentation et un programme d'entraînement intensif sont de même importance pour produire un accroissement statistiquement significatif de la force [25]. Des doses beaucoup plus importantes de 6 à $7000 \mathrm{mg}$ par semaine sont régulièrement administrées dans la pratique sportive, et des travaux récents ont montré que ces traitements supra-physiologiques produisaient l'accroissement de la taille et de la force musculaires le plus important, bien qu'augmentant aussi l'érytropoï̀se. On a aussi montré que des doses de cet ordre augmentaient la masse corporelle maigre, mais la preuve d'un effet sur la puissance aérobique et l'endurance est équivoque et d'autres investigations sont nécessaires [25].

Les profils d'utilisation abusive de stéroïdes peuvent être complexes, reflétant le souhait de minimiser les effets secondaires, d'échapper à la détection ou de répondre à quelque désir rituel. Les régimes les plus courants sont le régime périodique dans lequel les doses sont prises pendant 6 à 8 semaines suivies de 6 à 8 semaines d'abstinence, le régime pyramidal au cours duquel la dose de substance est lentement augmentée puis diminuée, et le régime de l'empilage dans lequel plusieurs stéroïdes ayant des propriétés pharmacocinétiques différentes sont pris simultanément. Il existe des versions féminines de ces programmes de dosage en particulier pour le régime de type périodique.

Les effets secondaires des stéroïdes anabolisants sont de plusieurs types. Il peut s'agir d'effets à court ou à long termes, aigus ou chroniques, réversibles ou non, masculinisant ou féminisant, généraux et/ou comportementaux [25]. Les effets à court terme des stéroïdes anabolisants sont largement connus en dépit de 50 ans d'utilisation abusive. Les stéroïdes anabolisants augmentent la rétention d'eau et de sel, ce qui amène une expansion du volume sanguin, mais les études sur les effets des stéroïdes sur la pression artérielle sont équivoques et la plupart de ces effets secondaires cardiovasculaires semblent être réversibles [62]. Toutefois, les anomalies ventriculaires, y compris l'hypertrophie, ont été reconnues chez des utilisateurs chroniques de stéroïdes dans plusieurs études récentes qui n'incluaient cependant qu'un nombre limité de sujets. L'abus de stéroïdes anabolisants entraîne une augmentation des taux san- 
guins de triglycérides et de cholestérol, et ceci est associé à une diminution des Lipoprotéines de Haute Densité (HDL) et à une élévation des Lipoprotéines de Faible Densité (LDL) $[25,49]$. Bien que ces effets soient réversibles, ils sont associés à un risque accru à la fois aigu et chronique de pathologie cardiovasculaire. Certaines de ces modifications pathologiques peuvent être facilement dépistées sous la forme de profil lipidique sanguin anormal, mais il existe aussi des pathologies fines de l'endothélium vasculaire qui contribuent à l'artériosclérose [18].

Certains des plus sérieux effets secondaires irréversibles des stéroïdes anabolisants sont associés aux cancers - principalement du foie, de la prostate et des reins. Les carcinomes hépatiques sont fortement associés à l'abus de la forme oralement active des stéroïdes 17-alpha-méthyl substitués, ces derniers étant aussi enclin à donner une jaunisse réversible [25].

Chez les hommes, l'abus de stéroïdes anabolisants est connu pour entraîner la suppression de la libération de LH et FSH, ce qui conduit à l'inhibition de la production de testostérone endogène. Cet état peut s'accompagner d'une atrophie des testicules, et d'une azoospermie réversible mais parfois irréversible. De fortes doses de substances pharmaceutiques prises régulièrement peuvent aussi entrainer des effets féminisants d'intensité modérée à forte, sous forme d'une gynécomastie. Le développement mammaire est du à «l'aromatisation» de la testostérone ou d'autres stéroïdes (mais pas de la nandrolone) en oestradiol [49].

Les caractères sexuels secondaires de type masculin représentent un effet secondaire des stéroïdes anabolisants chez la femme. Cela inclut le développement d'une pilosité faciale, la survenue d'une voix plus grave, un élargissement du pharynx, une croissance du clitoris et des modifications du squelette. Bien que l'aménorrhée soit souvent retrouvée chez les athlètes féminines pratiquant des sports d'endurance, l'aménorrhée induite par les stérö̈des peut être permanente et, sous sa forme réversible, peut être aussi associée à une infécondité.

L'acné constitue l'effet secondaire dermatologique des stéroïdes anabolisants le plus courant ; elle est particulièrement résistante aux traitements standards. Elle n'est pas toujours réversible.

Les effets secondaires endocriniens non gonadiques les plus courants des stéroïdes anabolisants sont constitués par une augmentation de la résistance à l'insuline et une hyperglycémie à jeun. Ces effets sont habituellement réversibles et la résistance à l'insuline ne conduit pas normalement à un diabète de type II [25].

L'abus de stéroïdes anabolisants amène des modifications comportementales, psychologiques et psychiatriques complexes pour lesquelles il existe un faible consensus [25,
42]. Beaucoup d'études sont contradictoires et ont été menées dans des groupes différents et non comparables soit d'athlètes soit de non-athlètes; et les mesures ont souvent porté sur des résultats ou des variables psychologiques différents. Quelques études indiquent que l'agressivité est augmentée chez les utilisateurs abusifs de stéroïdes anabolisants alors que d'autres études limitent cette augmentation à l'agressivité sexuelle, argumentant qu'un niveau élevé d'agressivité «contrôlée» est nécessaire à l'athlète victorieux [25]. Plusieurs investigateurs ont apporté la preuve d'une fréquence accrue de maladies mentales, telle que définies par les critères du DSM IV et de l'OMS, chez les utilisateurs abusifs de stéroïdes anabolisants, y compris la schizophrénie paranoïde, la manie et la dépression $[40,55]$. Tout aussi troublants sont les rapports montrant une dépendance physique et psychologique chez certains utilisateurs abusifs de stéroïdes anabolisants [11], ainsi que la survenue lors du sevrage, de troubles psychiatriques sévères pouvant amener dans quelques cas à des conduites criminelles et même au suicide [55].

Il est urgent d'avoir de plus nombreuses études sur les effets à long terme des stéroïdes anabolisants. Une étude menée en Finlande chez les haltérophiles utilisateurs abusifs de stéroïdes suggère que l'abus des stéroïdes anabolisants réduit la longévité : ils ont 4,6 fois plus de risque d'avoir une mort prématurée que les non utilisateurs abusifs [11].

Dans les chapitres suivant, on abordera et discutera l'action et les effets secondaires des stéroïdes anabolisants.

\section{TESTOSTERONE ET SUBSTANCES APPARENTEES}

La testostérone et ses dérivés synthétiques et semi-synthétiques ainsi que ses précurseurs immédiats sont dénommés hormones stéroïdes $\mathrm{C} 19$, une classification basée sur la structure du stéroïde androstane (Figure 1) [25]. Quand la testostérone est synthétisée dans le corps humain, son isomère épi-testostérone est aussi produit (Figure 1). Après la découverte et l'isolement de la testostérone en 1935, on a démontré que la testostérone possédait à la fois des propriétés anaboliques et androgéniques [25, 42]. Il s'est avéré quasiment impossible de séparer ces deux activités pharmacologiques $[25,42,86]$. Les stéroïdes, qui miment l'action de la testostérone, sont connus sous le nom de stéroïdes androgéniques anabolisants (SAA) ou simplement stéroïdes anabolisants (SA).

\section{Biosynthèse de la testostérone}

La testostérone (T) provient comme toutes les hormones stéroïdiennes du cholestérol, mais le précurseur immédiat en est l'androsténedione qui est converti en testostérone 
par un enzyme, la 17-beta-hydroxy-stéroïde déshydrogénasse (Figure 2) [25, 42, 75]. On a récemment découvert que le stéroïde anabolisant nandralone, qui est une 19-nortestostérone (Figure 1), peut être formé aussi bien chez l'homme que chez la femme à partir du 19-norandrosténédione [81]. Les implications de cette découverte sont discutées plus loin, elle ont aussi été abordées en détail par George [25] dans une revue précédente.

\section{Métabolisme de la testostérone}

La testostérone et son 19-nor dérivé, la nandrolone, sont convertis en étiocholanolone et androstérone et en leur 19nor dérivés respectifs par le système enzymatique hépatique de la 3alpha/3beta kéto-réductase [75,81]. La testostérone et la nandrolone peuvent être transformées par la 5alpha-réductase en leur dérivés dihydro respectifs, mais de ces deux, seule la testostérone peut être convertie en oestradiol par l'aromatase [75, 81, 83].

\section{Action de la testostérone et des stéroïdes anaboli- sants}

La testostérone produit ses effets au niveau cellulaire par :

1. diffusion à travers la membrane cellulaire et liaison au récepteur cytosolique aux androgènes ;

2. conversion dans la cellule cible en DHT (via l'enzyme 5-alpha-réductase);

3. conversion dans certaines cellules cibles en oestradiol via le système enzymatique aromatase et liaison ensuite au récepteur cytosolique à l'oestradiol.

Tous les mécanismes qui viennent d'être cités impliquent le transfert du complexe récepteur/hormone formé précédemment à une 'séquence impliquée dans la réponse hormonale' (hormone responsive element, HRE) dans le génome.

La testostérone et probablement aussi les stéroïdes anabolisants peuvent influencer l'activité cellulaire par :

4. liaison aux récepteurs de membrane de la surface cellulaire et leur activation, et par conséquent activation des systèmes du second messager ;

5. liaison à des sites modulateurs (facilitateurs ou atténuateurs) associés à certains récepteurs fonctionnels de la surface cellulaire comme le récepteur $\mathrm{GABA}_{\mathrm{A}}$ [29], plus particulièrement au niveau de son site de liaison à faible affinité pour les benzodiazépines [84].

La testostérone et les SA possèdent des effets androgéniques et anabolisants. Les modifications de la structure de la testostérone sont de 3 types majeurs : A, B et C. L'estérification du groupe 17-beta-OH avec une longue chaîne d'acides gras produit le type A. Les modifications du type
B sont constituées par une substitution alkyl en position 17 , alors que le type $\mathrm{C}$ implique l'addition de groupes chimiques habituellement en positions 1 à 5 . Les substitutions de type A produisent des préparations injectables ou retard, celles de type $\mathrm{B}$ confèrent une activité orale, tandis que les changements de type $C$ réduisent le métabolisme de ces stéroïdes. Une discussion complète de l'impact de ces modifications a été menée dans de nombreuses revues [24, $25,42,49,78]$. Bien que les modifications pré-citées induisent aussi des altérations du ratio de l'activité anabolisante/androgénique, un certain degré d'activité androgénique est toujours présent dans tous les stéroïdes anabolisants $[25,42]$.

\section{Modes d'administration}

Les modes et voies d'administration des stéroïdes anabolisants varient d'un groupe d' utilisateurs abusifs à l'autre. Les éléments déterminants en sont aussi bien la preuve empirique d'efficacité que les tentatives délibérées d'échapper à tout dépistage de l'abus par les autorités du sport et des athlètes. Des revues et analyses détaillées des régimes d'administration des stéroïdes anabolisants [25, 42] ont conduit à trois modes principaux qui peuvent être résumés comme suit :

a) Mode périodique : une période d'administration de la substance pharmaceutique est suivie d'une période équivalente d'abstinence ; puis la substance pharmaceutique est reprise de façon continue, par exemple 6 à 8 semaines avec/6 à 8 semaines sans, etc... Il peut exister des modes intriqués d'administration de la substance pharmaceutique et des pauses brèves ou vice-versa. Les profils d'abstinence peuvent être limités pour coïncider avec les compétitions dans le but d'échapper au dépistage, mais la stratégie globale est de réduire l'incidence d'effets secondaires [25, 43].

b) Mode pyramidal : ce mode est une variante du mode périodique dans laquelle la dose est graduellement augmentée sur une période pour arriver à un pic, puis ensuite progressivement réduite jusqu'à la fin de la période [25]. Ce régime à la réputation d'entraîner moins d'effets secondaires, tel qu'une réduction de l'entrain, dus à l'arrêt de la substance pharmaceutique, bien qu'il n'existe aucune preuve publiée de cela.

c) Mode d'empilage : ce mode implique l'utilisation de plus d'un stéroïde anabolisant au même moment. Un mode d'empilage implique l'utilisation simultanée à la fois d'un stéroïde administré par voie orale et d'un stérö̈de injectable. Des modes plus «sophistiqués» impliquent des profils intriqués d'administration faisant appel à de nombreux stéroïdes différents, chacun ayant des qualités pharmacologiques supposées différentes. Le but de cette technique est d'éviter ce qu'on appelle l'effet plateau ; c'est le dévelop- 
<smiles>CC12CCC(=O)C=C1CCC1C2CCC2(C)C1CC[C@@H]2O</smiles><smiles>C[C@]12CCC3C(CC[C@@H]4CC(=O)CC[C@]34C)C1CCC2O</smiles><smiles>CC12CCC3C(CC[C@@H]4C[C@H](O)CCC34C)C1CCC2=O</smiles><smiles>CC12CCC(=O)C=C1CCC1C2CCC2(C)C1CC[C@]2(C)O</smiles>

$17 u$-motmillestosterone

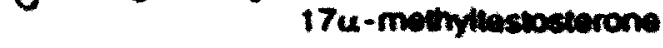

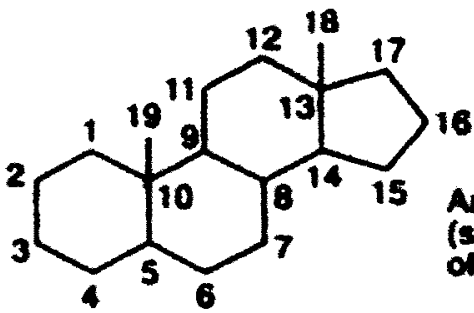
16 Androatene (showing the numbering of the certoon alomes<smiles>CC12CCC(=O)C=C1CCC1C2CCC2(C)C(O)CCC12</smiles><smiles>CC12CCC3C(CCC4=CC(=O)CCC43C)C1CCC2=O</smiles><smiles>CC12CC3C=NNC3C[C@H]1CCC1C2CCC2(C)C1CC[C@]2(C)O</smiles><smiles>CCCCC(=O)OC1CCC2C3CCC4=CC(=O)CCC4(C)C3CCC12C</smiles>

Figure 1 : Structure de la testostérone et de certains de ses dérivés synthétiques - adaptée de George [25]. 
<smiles>C[C@H]1[C@H]2CC[C@@]3(C)[C@@H]1CC=C2CC(O)CCC3(C)C</smiles>

dehydroandrostenedione(DHEA)

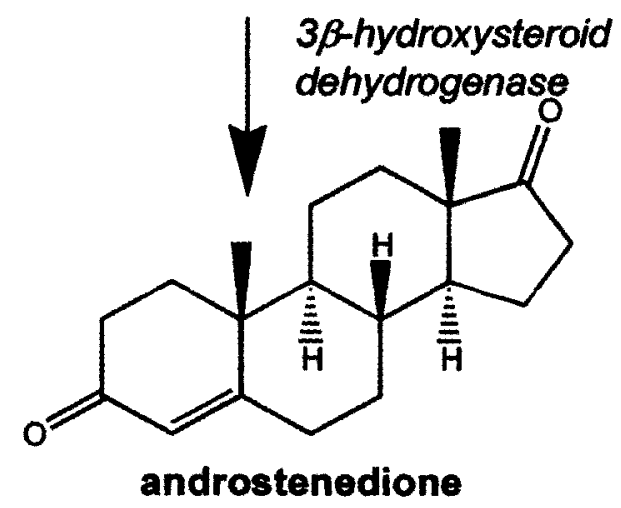

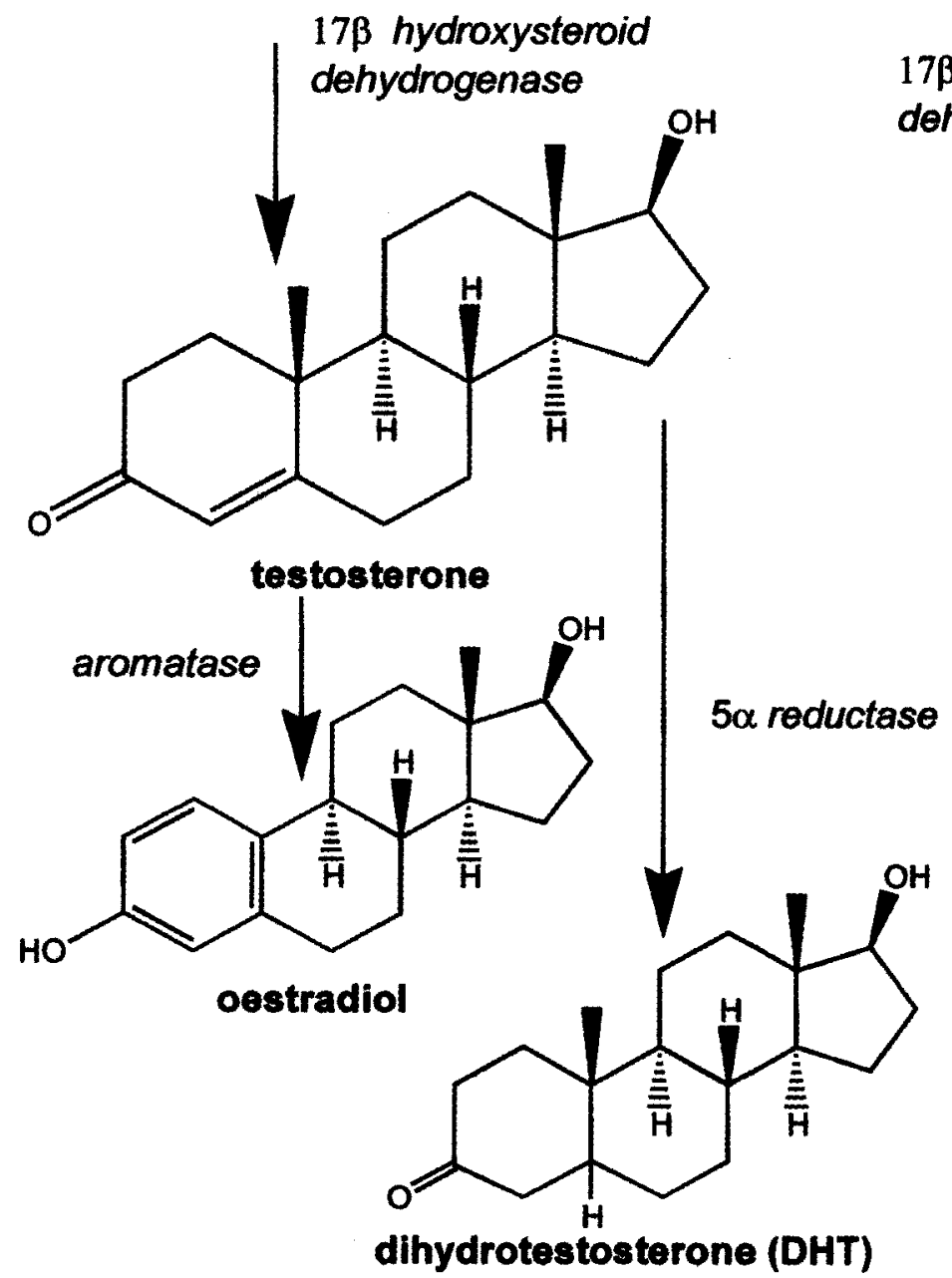

$\mathrm{HO}$<smiles>C[C@]12CC[C@H]3[C@@H]4CCC(O)CC4=CC[C@H]3[C@@H]1CCC2=O</smiles>

19 nordehydroandrostenedione (19nor DHEA)

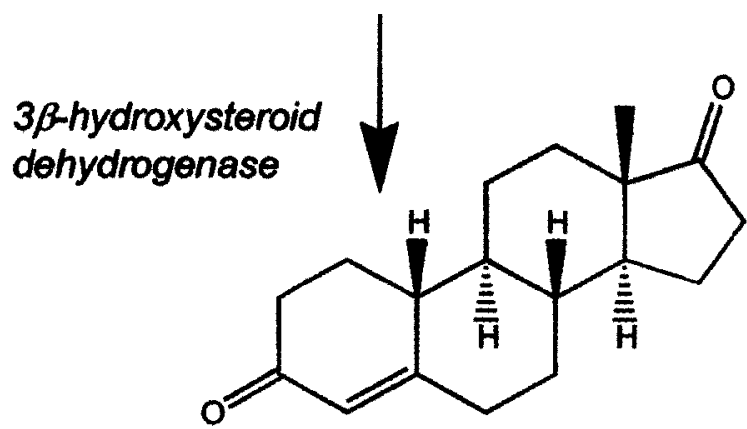

19 nor-androstenedione

$17 \beta$ hydroxysteroid dehydrogenase

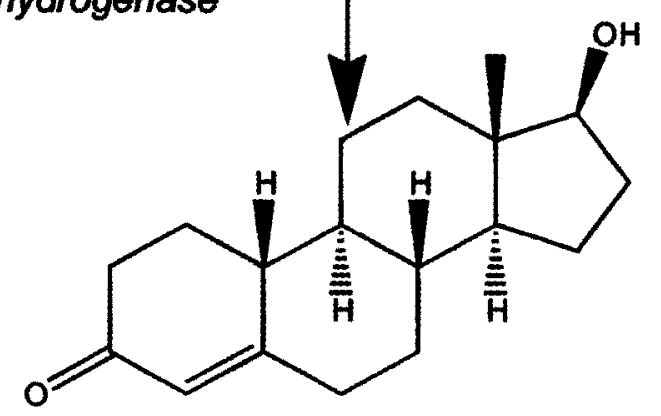

19 nor-testosterone

(Nandrolone)

$5 \alpha$ reductase

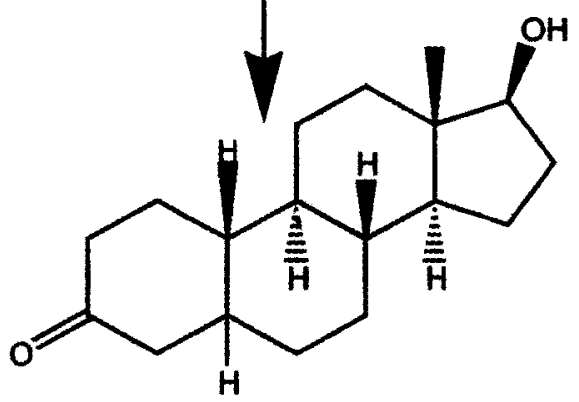

19 nor-dihydrotestosterone

Figure 2 : Biosynthèse de la testostérone et de ses dérivés - adaptée de George [25]. 
pement de la tolérance à une substance pharmaceutique en particulier. Ces programmes de 'super-empilage' sont «destinés» à rendre maximale l'occupation des sites récepteurs cytosoliques. Ceci est très douteux au plan pharmacologique puisque le nombre de récepteurs stéroïdiens intracellulaires à la testostérone/DHT est stable et tous sont probablement saturés dans les conditions physiologiques normales, ou tout au moins quand des doses thérapeutiques de stéroïdes anabolisants sont utilisées. L'efficacité de ce schéma d'administration a récemment été battue en brèche par d'autres [48]. Il est presque possible, cependant, que les doses supra-physiologiques administrées lors du mode d'empilage, et les taux plasmatiques subséquents de SA qui en résultent, puissent amener un déplacement des glucocorticoïdes $(\mathrm{GC})$ des récepteurs $\mathrm{GC}$, conduisant à une réduction du catabolisme protéique induit par les GC [25, 62]. De telles doses supra-physiologiques sont au moins 100 fois celles indiquées en thérapeutique en Europe et aux USA [63].

Une explication alternative est que le récepteur aux androgènes peut être augmenté par des doses supra-physiologiques de stéroïdes anabolisants [42], mais cela n'explique pas pourquoi des doses supra-physiologiques de testostérone sont capables d'inverser la perte musculaire présente chez les individus ayant des syndromes d'insensibilité aux androgènes [83].

A cette utilisation abusive de SA selon des profils et modes complexes, d'autres agents «anabolisants» non stéroïdiens peuvent être co-administrés. Les gonadotrophines chorioniques humaines (hCG) sont souvent utilisées abusivement par les athlètes pour stimuler la sécrétion naturelle de testostérone par le testicule [20] puisque cette hormone à un effet semblable à celui de la LH sur les cellules de Leydig testiculaires [26]. Un tel usage abusif de hCG est souvent employé pour contrecarrer la suppression de LH induite par l'administration chronique de stéroïdes anabolisants [26]. L'utilisation abusive de l'hormone de croissance (GH) est supposée réduire quelques uns des effets secondaires des stérö̈des anabolisants sur l'élévation lipidique, mais il n'existe aucune preuve publiée que cela soit vrai [25].

\section{Dosage}

Au Royaume-Uni, la dose standard d'entretien en testostérone chez l'homme varie entre 40 et $120 \mathrm{mg} / \mathrm{jour}$. Ceci est significativement moindre que les doses rapportées dans de nombreuses études cliniques/scientifiques sur les stéroïdes anabolisants $[25,42,63,78]$. Une courbe dose-réponse de l'effet de l'administration de stéroïdes anabolisants sur la masse maigre [21, 42] montre que de telles doses sont à la base de la courbe dose-réponse alors que l'effet maximum était atteint avec des doses dépassant $5000 \mathrm{mg} / \mathrm{semaine}$ !
Plusieurs études récentes ont montré d'une façon probante que ces doses massives, quand elles sont utilisées dans des conditions contrôlées, produisent des augmentations statistiquement significatives de la force musculaire et de la masse maigre comparées à la fois à un placebo et à des doses cliniques «normales» $[7,8]$.

\section{EST-CE QUE LES STEROIDES ANABO- LISANTS MARCHENT DANS LE SPORT ?}

Jusqu'à récemment, les résultats des effets de doses physiologiques de testostérone ou de stéroïdes anabolisants sur la force musculaire et les performances athlétiques étaient souvent conflictuels $[25,46]$. Les principales revues des données publiées depuis 1975 ont mis en lumière des erreurs cruciales dans les techniques expérimentales, conditions, critères d'évaluation, schéma statistique, doses et sujets utilisés dans les articles publiés durant cette période $[19,25,42,78]$. Certaines études anciennes n'ont pas de placebo, ou ne standardisent pas les doses, ou utilisent des moyens non reproductibles d'évaluation de la force $[19,65,78]$. Les auteurs de revue ont souvent confondus et comparés des études utilisant des hommes congénitalement ou pathologiquement hypogonadiques avec celles utilisant des hommes normaux ou entraînés [42].

En 1975, l'une des premières études scientifiquement contrôlée, croisée, démontra un léger mais significatif accroissement de la force chez les sujets prenant des doses cliniques de stéroïdes anabolisants comparés aux témoins [22]. Il fut établi en 1980 que de faibles doses de stéroïdes anabolisants produisaient un effet significatif sur la force musculaire seulement chez les athlètes entraînés qui s'exerçaient de façon régulière et intensive, et qui maintenaient un apport élevé en protéines $[34,80]$. Ainsi, ces aspects doivent tous être contrôlés dans toute étude de l'action des SA. L'exercice et l'entraînement induisent des augmentations de la masse et de la force musculaires qui sont souvent difficiles à différencier des effets des faibles doses de stéroïdes anabolisants. Une fois que les premiers expérimentateurs eurent établis que les doses thérapeutiques des stéroïdes anabolisants augmentaient bien la force musculaire, on se demanda alors si les doses élevées utilisées par les utilisateurs abusifs étaient nécessaires.

Il existe un problème éthique évident à soumettre des sujets normaux à des doses de stéroïdes anabolisants dépassant $5000 \mathrm{mg}$ par semaine. Toutefois, plusieurs groupes de chercheurs ont maintenant établi de façon non équivoque que des doses supra-physiologiques de stéroïdes anabolisants données à des athlètes entraînés au maximum, produisaient bien des augmentations significatives de la masse et de la force musculaires $[7,8]$. Les résultats des études biopsiques chez les haltérophiles indiquent que le nombre de fibres musculaires et la taille moyenne d'une 
fibre dans les muscles tels que le trapèze sont augmentés chez les utilisateurs abusifs de stérö̈des anabolisants comparés aux non utilisateurs abusifs [38]. L'effet des SA sur le muscle deltoïde de l'homme consiste en une augmentation de la taille des fibres musculaires de type II [31], et cette information vient s'ajouter aux études antérieures montrant que, au moins chez les mammifères, la testostérone augmente de façon différentielle la taille des muscles à contraction lente plutôt que celle des muscles à contraction rapide [42, 66]. Pour généraliser, la testostérone et, par conséquent, probablement tous les stéroïdes anabolisants, augmentent la puissance des muscles de la partie supérieure du corps plus que celle des muscles de la partie inférieure ; ils accroissent la force maximale volontaire et la puissance des jambes, mais ils ont peu d'effet sur la fatigabilité ou la tension du muscle [70].

Qu'est-ce qui détermine la réponse d'un adulte humain à la T et aux SA ? Chez les hommes adultes, il existe une variation ethnique considérable des concentrations sériques à la fois de la $\mathrm{T}$ et de la Sex Hormone Bending Globulin (SHBG) [33]. La SHBG est un déterminant majeur de la biodisponibilité de la $\mathrm{T}$ chez les hommes et les femmes [33]. On a retrouvé la testostérone totale liée et la $\mathrm{T}$ calculée libre plus basses chez les hommes Pakistanais que chez les «Caucasiens» ou les habitants des Caraïbes [33]. D'autres études sont nécessaires concernant ces résultats, dans le but d'élucider l'influence possible de la SHBG sur la capacité de réponse anabolique/androgénique.

Puisque les SA et la T sont connus pour produire la plupart de leurs effets via l'interaction avec une 'séquence génomique impliquée dans la réponse hormonale' (hormone responsive element, HRE), on peut s'attendre à ce que les polymorphismes de ce gène du Récepteur aux Androgènes (RA) puissent être corrélés à des différences dans la capacité de réponse générale aux SA, puisque au moins un groupe de chercheurs a démontré une relation entre la longueur augmentée du gène RA (en terme de nombres augmentés de répétition du trinucléotide $\mathrm{CAG}$ ) et une susceptibilité réduite au cancer de la prostate [33]. Cependant, une récente étude n'a montré qu'une faible association entre les polymorphismes de répétition des CAG du gène $\mathrm{RA}$ et la réponse à la $\mathrm{T}$ en terme de volume musculaire de la cuisse et de masse corporelle maigre [79]. Dans cette étude, les déterminants majeures de la capacité de réponse à la $\mathrm{T}$ étaient l'âge, la dose de $\mathrm{T}$ et la concentration sérique de l'antigène prostatique spécifique [79]. Toutefois, les nombres de RA doivent avoir une certaine influence sur la réponse aux SA chez les humains puisqu'une régulation positive du récepteur aux androgènes a été décrite dans le muscle exposé à des doses supra-physiologique de SA [68]. Cette discordance pourrait être résolue par l'observation récente que différents muscles comme le trapèze et le vaste externe contiennent des concentrations différents de RA et répondent différemment en terme de régulation positive du récepteur RA en réponse à l'entraînement et aux SA [39].

\section{EFFETS SECONDAIRES DES STEROI- DES ANABOLISANTS}

Les effets secondaires des stéroïdes anabolisants survenant suite à l'utilisation de doses cliniques de ces substances pharmaceutiques sont bien documentés et ont été récemment discutés en détail $[10,25,42,43,49]$. L'un des effets secondaires les plus courants est la rétention iodée qui donne naissance à une rétention d'eau puis à l'œdème [32, 62]. La rétention d'eau et une érythropoïèse augmentée contribuent à un accroissement du volume sanguin [42, 62] et aussi à l'augmentation du gain de poids qui survient après l'administration de stéroïdes anabolisants [32, 43]. Une grande partie de l'accroissement initial en taille et en volume du muscle qui survient après l'administration de SA est due à la rétention d'eau dans le tissu musculaire et non pas à une augmentation du contenu en protéines ou des fibres musculaires [32, 63]. Des effets secondaires plus sérieux, associés à une utilisation prolongée de SA, sont constitués par la jaunisse et le carcinome hépatique [28, 43]. Chez l'homme, les effets secondaires additionnels incluent la suppression de la sécrétion de $\mathrm{LH}$ et $\mathrm{FSH}$ conduisant à l'atrophie testiculaire [76], de sévères réductions ou un arrêt de la sécrétion de testostérone $[17,76]$ et une azoospermie [36]. Il existe un risque augmenté d'hyperplasie prostatique et peut être de carcinome prostatique [43]. Les effets secondaires chez les femme comprennent l'apparition de caractères sexuels secondaires mâles et la suppression des menstruations [25, 42, 43]. L'utilisation en pédiatrie de SA peut causer un arrêt de la croissance, via l'ossification des épiphyses, et aussi une puberté précoce chez les garçons $[10,25,43]$.

De nombreux types différents d'étude indiquent que les SA induisent des sensations d'énergie accrue, de bien être, d'amélioration de l'humeur, et d'agressivité accrue [10, 28]. D'une manière générale, les études et revues des effets comportementaux des SA se concentrent fortement sur les effets négatifs sur le comportement, qui ne peuvent être vus que pour les doses élevées administrées de façon abusive dans le sport [43]. On doit souligner que certains des effets négatifs observés après l'administration de SA peuvent être dus à l'effet de modifications induites dans la sécrétion d'autres hormones et non pas à l'action directe des SA eux mêmes [17]. Ceci est discuté plus loin avec les effets négatifs et addictifs des SA. Il n'existe aucune preuve dans la littérature scientifique ou clinique courante qu'une addiction aux SA survienne quand des SA sont administrés aux doses thérapeutiques [10]. 


\section{EFFETS SECONDAIRES DES STEROIDES ANABOLISANTS UTILISES ABUSIVEMENT DANS LE SPORT}

Certains des effets secondaires qui surviennent comme conséquence d'une utilisation abusive dans le sport sont simplement des amplifications de ceux précédemment décrits. Cependant, les doses supra-physiologiques communément prises par les athlètes tricheurs entraînent des effets secondaires supplémentaires, dont certains sont réversibles à l'arrêt de l'abus de stéroïdes, mais elles peuvent aussi entraîner des effets secondaires chroniques et irréversibles [42]. Les effets à long terme et les autres effets secondaires de l'utilisation abusive des stéroïdes anabolisants constituent un sujet important mais dont l'étude est sous-développée [25].

\section{Effets secondaires hypothalamo-pituitaires et endocrines}

L'administration aiguë et chronique de SA supprime les taux plasmatiques de gonadrophines [76], de testostérone [17] et d'hormones thyroïdiennes $\mathrm{T}_{3}$ et $\mathrm{T}_{4}$, mais a un effet insignifiant sur les hormones pituito-adrénaliennes [17]. Dans une étude récente, les modifications de l'agressivité survenant pendant l'administration de méthyl-testostérone sont corrélées spécifiquement aux augmentations de la thyroxine plasmatique libre [17]. Ces découvertes paradoxales concernant les hormones thyroïdiennes s'ajoutent au débat sur le rôle direct et indirect des SA et sur les modifications comportementales. Un effet secondaire plus sérieux des SA sur l'axe hypothalamo-pituitaire a aussi été récemment rapporté chez un homme pratiquant le body-building chez lequel il existait un hypogonadisme sévère, des taux plasmatiques de FSH et de LH indétectables mais une numération normale de spermatozoïdes [76]. Ces anomalies étaient présentes trois mois après l'arrêt de l'utilisation abusive de SA et, en fin de compte, le patient ne devint eugonadique qu'après un traitement de 200 microgrammes de LHRH pendant trois jours consécutifs [76]. Une gynécomastie survient parfois chez les athlètes abusant de doses de SA modérées à fortes [23], et la fréquence de cet effet secondaire augmente en cas d'utilisation abusive chronique [23]. L'augmentation de la taille de la poitrine est due à l'aromatisation de certains SA comme la $\mathrm{T}$, mais pas la nandrolone, en œstradiol [49]. C'est pour ces raisons que les utilisateurs abusifs s'administrent simultanément du tamoxifène, un antagoniste du récepteur à l'oestradiol, et aussi de l'amino-glutéthimide, un inhibiteur de l'aromatase [23].

On a montré que les stéroïdes anabolisants élevaient la résistance à l'insuline dans une étude [13], alors que dans un autre travail la nandrolone, mais pas la testostérone, augmentait la consommation de glucose dans le muscle
[35]. On a proposé que la capacité d'un SA à être aromatisé en oestradiol pouvait influencer ses effets sur l'homéostasie du glucose [35].

\section{Effets secondaires cardiovasculaires}

Les effets secondaires cardio-vasculaires sont peut être les conséquences de l'abus de stéroïdes anabolisants qui font l'objet des recherches les plus intenses. On a montré que les stéroïdes anabolisants augmentaient le volume sanguin, mais dans 8 des 9 cas revus par Rockhold [62], les sujets n'étaient pas des athlètes. Chez les athlètes traités par méthandionone, il y a une augmentation de $15 \%$ du volume sanguin total. Dans la plupart de ces études, y compris celle comprenant des athlètes, aucun contrôle du traitement n'est réalisé ; ainsi, l'accroissement du volume sanguin pourrait être la conséquence d'une érythropoïèse augmentée et non pas une augmentation directe du volume sanguin [62].

De nombreuses études et rapports documentés montrent que l'hypertension est liée à l'abus de stéroïdes anabolisants $[28,72,80]$, mais d'autres montrent des effets minimaux ou contradictoires, ou une absence d'effets sur la pression artérielle [62]. On a aussi suggéré que l'hypertension pourrait n'être induite que par certains types de SA [62]. La fonction ventriculaire des mammifères est altérée par l'administration de stéroïdes anabolisants [51], et les modifications pathologiques qui apparaissent sont semblables à celles trouvées à l'autopsie chez les sportifs ayant abusé de SA [2]. Chez les athlètes s'entraînant de façon intensive, les SA induisent un épaississement du ventricule gauche, une augmentation du volume diastolique final et de l'index de relaxation [80]. Une étude de Niemmen a rapporté, outre les résultats précédents, une hypertrophie cardiaque chez quatre jeunes haltérophilies. Deux de ces quatre haltérophiles avaient de plus des symptômes de défaillance cardiaque et l'un développa une thrombose massive des deux ventricules [2].

Les effets microvasculaires de l'abus des stéroïdes anabolisants ont été récemment explorés. Ebenbichler et al. [18] ont examiné la dilatation artériolaire par l'entremise du débit (FMD) chez 20 body-builders non fumeurs. Ils ont trouvé que la FMD était réduite chez les body builders comparés aux témoins non utilisateurs abusifs de SA, alors que la vasodilatation induite par le GTN (un anti-angineux) était réduite de façon non significative. Ces résultats sont corrélés à une diminution marquée des taux de cholestérol HDL chez certains athlètes. On peut conclure que l'abus de stéroïdes anabolisants est associé à un profil lipidique sanguin athérogène couplé à une dysfonction endothéliale menant à un risque augmenté d'athérosclérose.

Les stéroïdes anabolisants augmentent les taux sanguins de triglycérides et de cholestérol [2], et abaissent les taux san- 
guins de $\mathrm{HDL}$, principalement les fractions $\mathrm{HDL}_{2}$ et HDL 3, alors que la concentration en LDL est augmentée $[2,46]$. Le résultat de ces modifications du profil lipo-protéique sanguin est une augmentation du ratio cholestérol libre sur cholestérol lié au HDL [48]. La testostérone semble être, de tous les SA, le stéroïde ayant le moins d'effet sur le profil lipoprotéique sanguin [46]. La signification de ces découvertes est que l'étude américaine Framingham a montré qu'une simple réduction de 105 dans la concentration en HDL pouvait augmenter les risques d'une maladie cardiaque coronaire de $25 \%$. L'effet réducteur des SA sur le taux de HDL a été évalué à $25 \%$ dans une étude [15], mais peut atteindre $52 \%$ dans une autre étude [62]. Pour comparaison, chez les hommes normaux, $25 \%$ du cholestérol est sous forme HDL alors que chez les utilisateurs abusifs de SA seulement 7,8\% du cholestérol est lié aux HDL [25]. La plus grande réduction du cholestérol lié est observée avec les SA administrés par voie orale [46]. Toutefois, plusieurs études montrent que la réduction du cholestérol lié aux HDL peut être inversée quand l'abus de SA est arrêté $[15,45,62]$. Le temps de retour des taux à la normale varie de 3 à 5 semaines [45, 62] à 6 à 12 semaines [15]. Un abaissement des HDL sériques est un facteur de risque pour les maladies cardio-vasculaires et cérébro-vasculaires $[2,45,46]$, mais en même temps, les taux de cholestérol total peuvent rester inchangés $[27,43]$. Ceci peut conduire l'utilisateur abusif de SA, qui surveille son taux de cholestérol total, à une fausse impression de sécurité puisque l'athérosclérose associée à l'abus de SA peut ne pas être liée directement aux taux de cholestérol mais à d'autres modifications du métabolisme des lipides et aussi des triglycérides [27, 43]. En 1984, Goldman [28] a revu de nombreux cas d'athlètes en bonne santé âgés de moins de 40 ans qui avaient développé une maladie cardio-vasculaire alors qu'ils abusaient de stéroïdes anabolisants. Lukas [45] ne put trouver qu'un seul cas d'athlète mort de maladie cardio-vasculaire, alors qu'il abusait de stéroïdes anabolisants, dans les travaux publiés jusqu'en 1992. L'une des raisons pour ces faibles nombres d'athlètes mourant dans une situation apparemment à haut risque est que les utilisateurs abusifs de SA qui sont des athlètes pourraient être en meilleure santé que le reste de la population. Ceci pourrait repousser la survenue de modifications pathologiques à plus tard dans la vie [71]. Une analyse a fait état d'une série documentée de cas de sévères maladies cardio-vasculaires chez des utilisateurs abusifs de SA. Bien qu'il n'y eut qu'un seul décès, plusieurs cas sévères ne furent sauvés de la mort que par le recours à un traitement intensif et peut être par leur jeune âge [71]. L'analyse de toutes ces revues et rapports récents est compliquée par les différents modèles de substances pharmaceutiques et de doses utilisés, ainsi que par le style de vie des participants, en particulier le fait de fumer [25]. Cet aspect nécessite de plus amples recherches.
On peut attendre des modifications pathologiques précitées au niveau des HDL et de la fonction cardiaque qu'elles augmentent la coagulation sanguine et, par la suite, accroissent le risque de maladie cardiovasculaire [46]. Des études chez l'animal suggèrent que les SA augmentent bien le risque de troubles de la coagulation et d'embolie. Il existe une association entre l'abus de stéroïdes anabolisants et une agrégation plaquettaire augmentée [45]. Dans ces études, il y avait un cas de d'accident vasculaire cérébral induit par les stéroïdes anabolisants et trois cas d'embolie pulmonaire associés à l'abus de stéroïdes anabolisants, et un cas de thrombose veineuse [71].

\section{Effets hépatiques et rénaux}

Mêmes à doses cliniques, les stéroïdes anabolisants induisent une augmentation des taux d'enzymes hépatiques [22, 32], et à doses supra-physiologiques plus élevées, la jaunisse est un effet secondaire fréquent [43], bien que ces effets secondaires hépatiques soient habituellement réversibles à l'arrêt de l'abus de SA [43].

La jaunisse est de type intra-hépatique, choléstatique : elle résulte d'un débit réduit de la bile et de sa rétention accrue dans les capillaires biliaires des lobules hépatiques [69]. On a récemment rapporté un cas d'hépatite toxique résultant d'une utilisation abusive aiguë de doses supra-physiologiques de SA. Dans ce cas, la nécrose hépato-cellulaire fut confirmée par biopsie [69], et les anomalies disparurent après douze semaines de soins intensifs [69].

L'association entre l'administration de SA et le cancer du foie a été établie pour la première fois en 1965 [28]. Les stéroïdes anabolisants 17-alpha-alkylés sont les substances pharmaceutiques les plus fréquemment associées à cette condition [53]. L'abus de SA est aussi associé à des tumeurs de Wilms du rein [53].

\section{Effets sur la prostate}

Depuis de nombreuses années on soupçonne l'abus de SA d'être associé au carcinome prostatique. Une revue de trois importantes études cas-témoin a trouvé un lien puissant, bien que non sans équivoque, entre stéroïdes anabolisants élevés et cancer de la prostate, la $\mathrm{T}$ ayant la plus forte association avec ce carcinome [54]. Une étude antérieure et plus générale de suivi des effets secondaires et des effets adverses des SA, n'avait trouvé qu'un seul cas de cancer de la prostate [71]. Le risque de cancer de la prostate est augmenté chez l'homme par la présence d'un polymorphisme du gène RA [50]. Ce polymorphisme, incluant un nombre réduit de répétition du trinucléotide CAG, est plus courant chez les Afro-américains [50]. Ainsi, au moyen du génotypage, il peut être possible de déterminer à l'avance les individus plus susceptibles à cet effet secondaire. 


\section{Effets sur la fertilité}

L'administration de SA inhibe la libération de FSH et LH de la glande pituitaire antérieure chez l'homme et la femme $[37,47]$. Des doses de stéroïdes anabolisants aussi faibles que $15 \mathrm{mg}$ par jour pendant deux mois peuvent causer jusqu'à $75 \%$ de réduction de la concentration de sperme ainsi qu'une azoospermie [36]. La sévérité de la suppression des gonadotrophines et les effets anti-fertilité subséquents dépendent de la dose et de la durée de l'abus de SA [47]. La normalisation de la numération du sperme après abus de SA peut être accélérée par traitement par hCG [26, 74] ou par LH-RH [76]. Toutefois, un retour de la numération de sperme à la normale ne signifie pas nécessairement que les spermatozoïdes produits soient féconds [43]. Les preuves que les SA puissent causer une infertilité permanente sont équivoques et une étude n'a retrouvé aucune preuve d'effets chroniques chez des adultes jeunes [71].

\section{Effets sur la libido et le comportement}

La libido des hommes et des femmes est influencée par la testostérone, mais les études des effets de la testostérone et des SA sur la libido sont compliquées par l'utilisation de patients hypogonadiques plutôt que de sujets eugonadiques. Plusieurs études ont indiqué l'importance de la $\mathrm{T}$ dans le maintien d'aspects clés de la sexualité masculine tels que la fonction érectile et les fantasmes sexuels [4, 5]. Cependant, dans une étude, des doses de $500 \mathrm{mg}$ par semaine sur une période de 14 semaines n'ont entraîné aucune augmentation de la libido masculine [85].

Les preuves sont contradictoires en ce qui concerne les effets des SA sur le comportement. Alors qu'au moins un auteur n'a retrouvé aucune relation entre administration de SA et augmentation de l'agressivité [3], une autre étude de suivi postérieure rapporte des effets sujets à caution et équivoques des SA sur le comportement [77]. Plusieurs recherches ont rapporté des sensations accrues d'énergie, de l'agressivité, et une meilleure humeur chez les athlètes abusant de stéroïdes anabolisants $[6,57,64]$. En utilisant des doses élevées chez des volontaires normaux, deux études ont montré que l'hostilité, l'irritabilité, l'euphorie, la conduite et l'éveil sexuels étaient augmentés par la SA [15, 57], et une autre étude a rapporté que les comportements à type de réponse agressive étaient accrus par les SA [41]. Cependant, quand des doses supra-physiologiques de stéroïdes anabolisants sont utilisées chez des hommes normaux, une augmentation de l'agressivité n'est pas toujours observée [43]. La recherche de la cause sous-jacente de ces modifications a récemment porté sur l'observation des changements endocriniens plasmatiques/sériques [17] et des modification de la composition du fluide cérébro-spinal (CSF) [16]. Il est intéressant de noter que certaines modifications du comportement ont été associées à des réductions des taux d'hormones thyroïdiennes [17].

\section{Effets sur la santé mentale}

On a montré que les effets des stéroïdes anabolisants sur l'état mental de patients et d'utilisateurs abusifs étaient aussi équivoques que les effets sur le comportement. Les femmes souffrant d'une dépression clinique du post-partum ont des taux de testostérone bas [1]. Les SA ont des effets anti-dépresseurs chez plusieurs groupes de patients déprimés [58,60,67], y compris chez ceux souffrant du VIH [60], et les SA ont aussi été ajoutés au traitement de certains patients présentant des dépressions résistantes aux traitements $[58,67]$.

A l'opposé, plusieurs cas de manie ont été rapportés chez les utilisateurs abusifs de stéroïdes anabolisants $[40,55]$, tandis que des utilisateurs abusifs de SA en dépression et qui tentaient d'arrêter les stérö̈des anabolisants développèrent une manie quant ils furent traités par anti-dépresseurs $[40,77]$.

Il est communément accepté que l'agressivité et l'hostilité sont des composantes importantes de la personnalité des sportifs modernes. Cependant, ceci peut n'être vrai que pour certains sports tel que la lutte [61]. Alors que certaines études ont montré une augmentation générale de l'hostilité et de l'agressivité avec l'abus de SA [57], d'autres ont amené la preuve d'hostilité, de violence et d'abus physique de partenaire par des individus utilisateurs de stéroïdes anabolisants de façon abusive [12]. Toutefois, quand on utilise de faibles doses de SA (110 à $550 \mathrm{mg}$ par semaine), aucune augmentation de l'hostilité n'a pu être détectée [85]. Au moins 8 cas bien documentés de suicide ont été reliés à l'arrêt de l'abus de SA [73], alors que l'analyse des taux de testostérone du CSF chez 46 patients suédois ayant tentés de se suicider montra des taux de testostérone plus bas que chez les patients agressifs/violents d'autres études [30]. L'usage abusif de SA semble aussi augmenter le risque de faire l'expérience de la violence physique, y compris le meurtre [54].

\section{Addiction et dépendance}

Plusieurs études dans les années 80 ont démontré une addiction et une dépendance chez les hommes utilisateurs abusifs de SA [10, 40], mais une seule chez les femmes [14]. Un désordre dépendant de l'hormone stéroïde sexuelle a été décrit [40], et les facteurs biochimiques, physiologiques et psychologiques relatifs à une dépendance aux SA ont été évoqués [9] et résumés [10]. Récemment, un syndrome dysmorphique musculaire a été suggéré comme l'une des causes possibles de dépendance [56], et celui-ci peut avoir plusieurs formes d'expression et peut être lié à l'anorexie chez les hommes [52]. La peur d'une «décons- 
truction musculaire» suite à l'arrêt de l'abus de stéroïdes anabolisants a été proposée comme l'une des composantes psychologiques possibles de l'addiction au SA [25]. Dès 1989 , un lien entre abus de SA et crime sérieux était suggéré [44]. La personnalité sous-jacente des utilisateurs abusifs [6] et dépendants [9] de SA a aussi été récemment analysée, et une association entre abus de SA et conditionnement de l'image sociale a été proposée [82]. Des facteurs de la personnalité comme le narcissisme et une faible empathie (partage des émotions d'autrui) ont été associés à l'abus de SA dans au moins une étude [59]. Plusieurs auteurs ont insisté sur le fait que la majorité des athlètes et des body-builders qui abusent de SA ne rapportent pas de symptômes psychiatriques majeurs $[6,45]$ ou se retiennent de le faire [45]. Il n'est pas non plus nécessairement vrai qu'un utilisateur abusif de SA qui se sent plus agressif et qui rapporte de lui-même plus d'agression, se comportera violemment ou développera un trouble mental [6].

\section{Longévité}

Ce n'est qu'au cours des 5 dernières années que des tentatives sérieuses ont eu lieu pour examiner l'influence des stéroïdes anabolisants sur la mortalité. Dans une étude portant sur 62 haltérophiles d'élite finlandais, une mort prématurée était retrouvée 4,6 fois plus souvent chez les utilisateurs abusifs de SA que dans la population témoin qui n'abusait pas de SA [53]. Un examen ultérieur des sujets de cette étude montra que l'haltérophilie par elle-même n'augmentait pas les taux de mortalité ; c'est pourquoi l'augmentation de la mortalité dans l'étude précédente fut attribuée à l'effet des SA [53, 54].

\section{CONCLUSION}

Les SA ont été utilisés de façon abusive par les athlètes et dans la pratique sportive depuis au moins 50 ans [86]. En dépit de nombreuses études détaillées démontrant plusieurs effets secondaires aigus et chroniques, tant physiques que mentaux, des stérö̈des anabolisants, la plupart de ces effets secondaires semblent être réversibles [45]. Il est urgent de réaliser plus d'études pour savoir qui est susceptible d'être plus sensible aux effets secondaires et quels sont les effets de ces substances sur la mortalité. En dehors du sport, il est nécessaire d'être capable de traiter l'abus social et cosmétique de ces substances pharmaceutiques. Reste à savoir si le Sorcier Anabolisant reviendra un jour pour rompre l'ensorcellement anabolique.

\section{REFERENCES}

1. ALDER E.M., COOK A., DAVIDSON D., WEST C. BANCROFT J. : Hormones, mood and sexuality in lactating women. Br. J. Psychiatry, 1986, $148: 74-79$.

2. ALEN M., RAHKILA P. : Anabolic-androgenic steroid effects on endocrinology and lipid metabolism in athletes. Sports Med., $1988,6: 327-332$.

3. ARCHER J. : The influence of testosterone on human aggression. Br. J. Psychol., 1991, 82 : 1-28.

4. BAGATELL C.J., BREMNER W.J. : Androgens in men uses and abuses New Engl J. Med., 1996, 334 : 707-714.

5. BAGATELL C.J., HEIMANN J.R., RIVIER J.E. : Effects of endogenous testostrone and estradiol on sexual behaviour in normal young men. J. Clin. Endocrinol. Metab., 1994, 78 : 713-716.

6. BAHRKE M.S. : Psychological effects of endogenous testosterone and anabolic-androgenic steroids. In : Yesalis C. ed. Anabolic Steroids in sport and exercise. Champaign Il, Human Kinetics Publishers, 2000 : 94-106.

7. BHASIN S., STORER T.W., BERMAN N. et al. : The effects of supraphysiologic doses of testosterone on muscle size and strength in normal men. N. Engl. J. Med., 1996, $335: 1$-7.

8. BHASIN S., WOODHOUSE L., STORER T.W. : Proof of the effect of testosterone on skeletal muscle. J. Endocrinol., 2001, $170: 27-38$.

9. BROWER K.J. : Anabolic steroids : potential for physical and psychological dependence. In : Yesalis C. ed. Anabolic steroids in sport and exercise. Champaign Il, Human Kinetics Publishers, $2000: 279-304$.

10. BROWER K.J. : Anabolic steroid abuse and dependence. Curr. Psychiatry Reports, 2002, 4 : 377-387.

11. BROWER K.J., BLOW F.C., YOUNG J.P., HILL E.H. : Symptoms and correlates of anabolic steroid dependence. $\mathrm{Br} . \mathrm{J}$. Addict., 1991, $86: 759-768$.

12. CHOI P.Y.L, POPE H.G. : Violence towards women and illicit androgen-anabolic steroid use. Ann. Clin. Psychiatry, 1994, 6 : 21-25.

13. COHEN J.C., HICKMAN R. : Insulin resistance and diminished glucose tolerance in powerlifters ingesting anabolic steroids. $\mathbf{J}$. Clin. Endocrinol. Metab., 1987, 64 : 960-963.

14. COPELAND J., PETERS R., DILLON P. : Anabolic-androgenic steroid dependence in a woman. Austr. N.Z. J. Psychiatry, 1998, $32: 589-591$.

15. COSTILL D.L., PEARSON D.R., FINK W.J. : Anabolic steroid use among athletes. Changes in HDC-C levels. Phys. Sports Med., 1984, 12 : 113-117.

16. DALY R.C., SU T-P., SCHMIDT P.J., PICKAR D., MURPHY D.L., RUBINOW D.R. : Cerebrospinal fluid and behavioural changes after methyltestosterone administration. Arch. Gen. Psychiatry, 2001, $58: 172-177$.

17. DALY R.C., SU T-P., SCHMIDT P.J., PAGLIARO M., PICKAR D., RUBINOW D.R. : Neuroendocrine and behavioural effects of high-dose anabolic steroid administration in male normal volunteers. Psychoneuroendocrinology, 2003, 28 : 317-331. 
18. EBENBICHLER C.F., STURM W., GANZER H. et al. : Flowmediated, endothelium dependent vasodilation is impaired in male body builders taking anabolic-androgenic steroids. Atherosclerosis, 2001, $158: 483-490$.

19. ELASHOFF J.D., JACKNOW A.D., SHAIN S.G., BRAUNSTEIN G.D. : Effects of anabolic-androgenic steroids on muscular strength. Ann. Int. Med., 1991, 115 : 387-393.

20. EVANS N.A. : Gym and tonic : a profile of 100 male steroid users. Br. J. Sports Med., 1997, 31 : 54-58.

21. FORBES G.B. : The effect of anabolic steroids on lean body mass. The dose response curve. Metabolism, 1985, $34: 571-573$.

22. FREED D.L.J., BANKS A.J., LONGSON D., BURLEY D.M. : Anabolic steroids in athletes: cross-over double blind trial in weightlifters. Br. Med. J., 1975, 2 : 471-473.

23. FRIEDL K.E., YESALIS C.E. : Self-treatment of gynaecomastia in body builders who use anabolic steroids. Phys. Sports Med.. $1989,17: 67-79$.

24. GEORGE A.J. : Drugs in Sport - chemists $v$ cheats -a score draw ! Chem. Rev., 4 : 10-14.

25. GEORGE A.J. : Anabolic steroids. In : Mottram D.R. ed. Drugs in Sport $3^{\text {rd }}$ Edition. London, E. \& F. Spon, 2003, 138-188.

26. GILL G.V. : Anabolic steroid induced hypogonadism treated with human chorionic gonadotrophins. Postgrad. Med. J., 1998, $74: 45-46$.

27. GLAZER G. : Arthrogenic effects of anabolic steroids on serum lipid levels. Arch. Int. Med., 1991, 151 : 1925-1933.

28. GOLDMAN B. : Death in the locker room : Steroids and sports. London, Century Publishing, 1984.

29. GUO Z., BENTEN W.P., KRUCKEN J., WUNDERLICH F. : Nongenomic testosterone calcium signalling : genomic actions in androgen receptor- free macrophages. J. Biol. Chem., 2002, 277 : 29600-29607.

30. GUSTAVSSON G., TRASKMAN-BENDZ L., DEE HIGLEY J., WESTRIN $\AA$. : CSF testosterone in 43 male suicide attempters. Europ. Neuropsychopharmacology, 2003, $13: 105-109$.

31. HARTGENS D., VAN STRAATEN H., FIDELDIJ S., RIETJENS G., KEIZER H.A., KUIPERS H. : Misuse of androgenicanabolic steroids and human deltoid muscle fibers : differences between polydrug regimens and single drug administration. Europ. J. Appl. Physiol., 2002, 86 : 233-239.

32. HAUPT H.A., ROVERE G.D. : Anabolic steroids : A review of the literature. Am. J. Sports Med., 1984, $12: 469-484$.

33. HEALD A.H., IVISON F., ANDERSON S.G., CRUICKSHANK K., LAING I., GIBSON J.M. : Significant ethnic variation in total and free testosterone concentration. Clin. Endocrinol., 2003, $58: 262-266$.

34. HERVEY G.R., KNIBBS A.V., BURKINSHAW L. et al. : Effects of methandione on the performance and body composition of man undergoing athletic training. Clin. Sci., 1981, 60 : 457-461.

35. HOBBS C.J., JONES R.E., PLYMATE S.R. : Nandrolone, a 19nortestosterone, enhances insulin dependent glucose uptake in normal men. J. Endocrinol. Metab., 1996, 81 : 1582-1585.
36. HOLMA P.K. : Effects of an anabolic steroid (methandienone) on spermatogenesis. Contraception, 1979, 15:151-162.

37. JAROW J.P., LIPSCHULTZ L.I. : Anabolic steroid induced hypogonadotrophic hypogonadism. Am. J. Sports Med., 1990, 18: 429-431.

38. KADI F., ERIKSSON A., HOLMNER S., BUTLER-BROWNE G., THORNHILL L.E. : Cellullar adaptation of the trapezius muscle in strength trained athletes. Histochem. Cell Biol., 1999, $111: 189-195$.

39. KADI F., BONNERUND P., ERIKSSON A., THORNELL L.E. : The expression of androgen receptors in human neck and limb muscles : effects of training and self-administration of androgenic-anabolic steroids. Histochem. Cell Biol., 2000, 113 : 25-29.

40. KASHKLIN K.B., KLEBER H.D. : Hooked on hormones ? An anabolic steroid addiction hypothesis. J. Amer. Med. Assoc., $1989,262: 3166-3170$

41. KOURI E.M., LUKAS S.E., POPE H.G., OLIVA P.S. : Increased aggressive responding in male volunteers following the administration of gradually increasing doses of testosterone cypionate. Drug Alcohol Depend., 1995, 40 : 73-79.

42. KUHN C.M. : Anabolic Steroids. Endocrinol. Rev., 2002, 13 : 411-434.

43. KUTSCHER E.C., LUND B.C., PERRY P.J. : Anabolic Steroids -A review for the clinician. Sports Med., 2002, 32 : 285-296.

44. LUBELL A. : Does steroid abuse cause - or excuse - violence ? Phys. Sport Med., 1989, 17 : 176-185.

45. LUKAS S.E. : Current perspectives on anabolic-androgenic steroid abuse. Trends Pharmacol. Sci., 1993, 14 : 61-68.

46. LOMBARDO J.A., HICKSON P.C., LAMB D.R. : Anabolic/androgenic steroids and growth hormone. In : Lamb D.R., Williams M.H. eds. Perspectives in Exercise Science and Sports Medicine, Vol. 4 : Ergogenics - Enhancement of Performance in Exercise and Sport. New York, Brown and Benchmark, 1991, 249-278.

47. MCINDOE J.H., PERRY P.J., YATES W.R. et al : Testosterone suppression of the HPT axis. J. Invest. Med., 1997, $45: 441-447$.

48. MILLAR A.P. : Licit steroid use - hope for the future. Br. J. Sports Med., 1994, 28 : 79-83.

49. MOTTRAM D.R., GEORGE A.J. : Anabolic steroids. Clin. Endocrinol. Metab., 2000, 14 : 55-69.

50. NELSON K.A., WHITE J.S. : Androgen receptor CAG repeats and prostate cancer. Am. J. Epidemiol., 2002, 155 : 883-890

51. NIEMINEN M.S., RAMO M.P., VIITASALO M. et al. : Serious cardiovascular side effects of large doses of anabolic steroids in weight lifters. Europ. Heart J., 1996, 17 : 1576-1583.

52. OLIVARDIA R., POPE H.G., HUDSON J.L. : Muscle dysmorphia in male weightlifters : a case control study. Am. J. Psychiatry, 2000, $157: 1291-1296$.

53. PARSINNEN M., KINJALA U., VARTIAINEN E., SARNA S., SEPPALA T. : Increased premature mortality of competitive powerlifters suspected to have used anabolic agents. Int. J. Sports Med., 2000, 21 : 225-227.

54. PARSSINEN M., SEPPALA T. : Steroid use and long-term 
health risks in former athletes. Sports Med., 2002, 32 : 83-94.

55. POPE H.G., KATZ D.L : Psychiatric and medical effects of anabolic-androgenic steroid use. Arch. Gen. Psychiat., 1994, 51 : 375-382.

56. POPE H.G., GRUBER A.J., CHOI P., OLIVARDIA R., PHILLIPS K.A. : Muscle dysmorphia. Psychosomatics, 1997, 38 : 548-557.

57. POPE H.G., KOURI E.M., HUDSON J.I. : Effects of supraphysiological doses of testosterone on mood and aggressionin normal men. Arch. Gen. Psychiatry, 2000, 57 : 133-140.

58. POPE H.G., COHANE G.H., KANAYAMA G., SIEGEL A.J., HUDSON J.I. : Testosterone gel supplementation for men with refractory depression : a randomised placebo-controlled trial. Am. J. Psychiatry (in press).

59. PORCERELLI J.H., SANDLER B.A. : Narcissism and empathy in steroid users Ann. J. Psychiatry, 1995, 152 : 1672-1674.

60. RABKIN J.G., WAGNER G.J., RABKIN R. : A double blind, placebo controlled trial of testosterone therapy for HIV-positive men with hypogonadal symptoms. Arch. Gen. Psychiatry, 2000, $57: 141-147$.

61. RAGLIN J.S. : Psychological factors in sport performance. Sports Med., 2001, 31 : 875-890.

62. ROCKHOLD R.W. : Cardiovascular toxicity of anabolic steroids. Ann. Rev. Pharmac. Tox., 1993, 33 : 497-520.

63. ROGOL A.D., YESALIS C.E. : Anabolic-androgenic steroids and athletes: What are the issues ? J. Endocrinol. Metab., 1992, $74: 465-469$.

64. RUBINOW D.R., SCHMIDT P.J. : Androgens, brain and behaviour. Am. J. Psychiatry, 1996, 153 : 974-984.

65. RYAN A.J. : Anabolic steroids are fool's gold. Fed. Proc., 1981, $40: 2682-2688$

66. SACHS B.D., LEIPHEIMER R.E. : Rapid effect of testosterone on striated muscle activity in rats. Neuroendocrinology, 1988, 48: $453-458$.

67. SEIDMANN S.N., RABKIN J.G. : Testosterone replacement therapy for hypogonadal men with SSRI-refractory depression. J. Affect. Disord., 1998, 48 : 157-161.

68. SHEFFIELD-MOORE M., URBAN R.J., WOLF S.E. et al. : Short-term oxandrolone administration stimulates net muscle protein synthesis in young men. J. Clin. Endocrinol. Metab., 1999, $84: 27052711$.

69. STIMAC D., MILLI S., DINTINJANA R.D., KOVAC D., RISTI $\mathrm{S}$. : Androgenic/Anabolic steroid induced toxic hepatitis J. Clin. Gastroenterol., 2002, 35 : 350-352.

70. STORER T.W., MAGLIANO L., WOODHOUSE L. et al. : Testosterone dose-dependently increases maximal voluntary strength and leg-power, but does not affect fatigability or specific tension. J. Endocrinol. Metab., 2003, 88 : 1478-1485.

71. STREET C., ANTONIO J., CUDLIPP D. : Androgen use by athletes: A re-evaluation of the health risks. Canad. J. Appl. Physiol., 1996, 21 : 421-440.

72. SULliVAN M.L., MARTINEZ C.M., GENNIS C. et al. : The cardiac toxicity of anabolic steroids. Prog. Cardiovasc. Dis., 1998, $41: 1-15$.
73. THIBLIN I., LINDQUIST O., RAJS J. : Cause and manner of death among abusers of anabolic androgenic steroids. J. Forensic Sci., 2000, $45:$ 16-23.

74. TUREK P.J., WILLIAMS R.H., GILLBRAUCH J.H. et al : The reversibility of anabolic steroid induced azoospermia. J. Urol., $1995,153: 1628-1630$.

75. UEKI M., OKANA M. : Doping with naturally occurring steroids. J. Toxicol. Toxin. Rev., 1999, $18: 177-195$.

76. VAN BREDA E., KEIZER H.A., KUIPERS H., WOLFFENBUTTEL B.H.R. : Androgenic anabolic steroid use and severe hypothalamic-pituitary dysfunction: a case study. Int. J. Sports Med., 2003, 24 : 195-196.

77. WILLIAMSON P.J., YOUNG A.H. : Psychiatric effects of androgenic and anabolic-androgenic steroid abuse in men : a brief review of the literature. J. Psychopharmac., 1992, $6: 20-26$.

78. WILSON J.D. : Androgen abuse by athletes. Endocrinol. Rev., 1988, $9: 181-199$.

79. WOODHOUSE L.J., REISZ-PORSZASZ S., JAVANBAKHT M. et al : Development of models to predict anabolic response to testosterone administration in healthy young men. Am. J. Physiol., 2003, 284 : 1009-1017.

80. WRIGHT J.E. : Anabolic steroids and athletics. Exerc. Sport Sci. Rev., $1980,8: 149-202$.

81. WRIGHT F., BRICOUT V., DOUKANI A., BONGINI M. : Nandrolone et nor-stéroïdes : substances endogènes ou xénobiotiques? Sci. Sports, 2000, $15: 111-124$.

82. WROBLEWSKA A.M. : Androgenic-anabolic steroids and body dysmorphia in young men. J. Psychosom. Res., 1997, 42 : 225234.

83. WU F.C.W. : Endocrine agents of anabolic steroids. Clin. Chem., $1997,43: 1289-1292$.

84. YANG P., JONES B.L., HENDERSON L.P. : Mechanisms of anabolic androgenic steroid modulation of alpha(1)beta(3)gamma(2L) GABA $\mathrm{G}_{\mathrm{a}}$ receptors. Neuropharmacology, 2002, $43: 619-633$.

85. YATES W.R., PERRY P.J., MACINDOE J., HOLMAN T. ELLINGRAD V. : Psychosexual effects of 3 doses of testosterone cycling in normal men. Biol. Psychiatry, 1999, $45: 254-260$

86. YESALIS C., COURSON S., WRIGHT J. : History of anabolic steroid use in sport and exercise. In : Yesalis C. ed. Anabolic Steroids in sport and exercise. Champaign Il, Human Kinetics Publishers, 2000 : 54-56.

Communication au $\mathrm{XI} \mathrm{X}^{\circ}$ Congrès de la Société d'Andrologie de Langue Française, Genève, 12-14 decembre 2002.

Manuscrit reçu : juillet 2003 ; accepté septembre 2003. 